\title{
Influence of Capping on the Atomistic Arrangement in Palladium Nanoparticles at Room Temperature
}

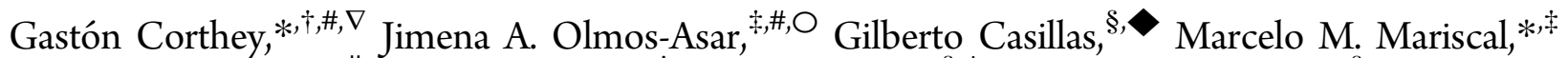

Sergio Mejía-Rosales, ${ }^{\|}$Julio C. Azcárate, ${ }^{\dagger}$ Eduardo Larios, ${ }^{\S, \perp}$ Miguel José-Yacamán, ${ }^{\S}$

Roberto C. Salvarezza, ${ }^{\dagger}$ and Mariano H. Fonticelli* ${ }^{\dagger}$

${ }^{\dagger}$ Instituto de Investigaciones Fisicoquímicas, Teóricas y Aplicadas (INIFTA), Universidad Nacional de La Plata, CONICET, Sucursal 4 Casilla de Correo 16 (1900), La Plata, Argentina

${ }^{\ddagger}$ INFIQC, CONICET, Departamento de Matemática y Física, Facultad de Ciencias Químicas, Universidad Nacional de Córdoba (XUA5000), Córdoba, Argentina

${ }^{\S}$ Department of Physics and Astronomy, University of Texas at San Antonio, One UTSA Circle, San Antonio, Texas 78249, United States

"Center for Innovation, Research and Development in Engineering and Technology and Centro de Investigación en Ciencias Físico Matemáticas (CICFIM), Facultad de Ciencias Físico-Matemáticas, Universidad Autónoma de Nuevo León, 66450 San Nicolás de los Garza, Nuevo León, México

${ }^{\perp}$ Departamento de Ingeniería Química, Universidad de Sonora, 83000 Hermosillo, Sonora, México

\section{Supporting Information}

ABSTRACT: The role that protecting molecules have on the way that palladium atoms arrange themselves in nanoparticles prepared at room temperature was studied by the analysis of aberration-corrected scanning transmission electron microscopy images and atomistic Langevin dynamics simulations. It was found that the arrangement of $\mathrm{Pd}$ atoms is less ordered in thiolate-protected nanoparticles than in amine-protected ones. The experimental and theoretical data showed that the disorder in $\sim 3 \mathrm{~nm}$ thiolate-protected particles is promoted by the strong S-Pd bond in the sulfide layer that surrounds the nanoparticles.

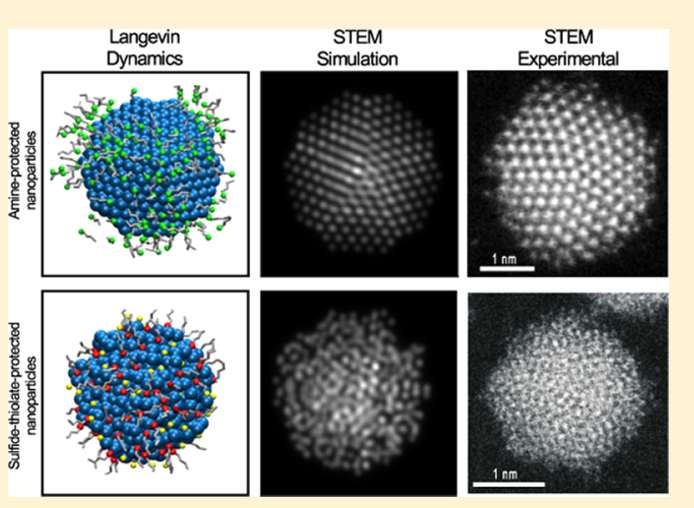

\section{INTRODUCTION}

The unique properties of thiolate self-assembled monolayers (SAMs) have provoked great interest in these assemblies, especially for their role in the stability and interfacial properties of thiolate-protected noble-metal nanoparticles (NPs). ${ }^{1,2}$ Gold nanoparticles are among the most studied metal nanoparticles, to the point of being considered the de facto model system in this field of research. ${ }^{3,4}$ However, some properties of other metals can be very different from those of gold, and the case of palladium is an example that fulfills this statement. For instance, the adsorption of alkanethiols on planar palladium surfaces produces a sulfide layer (with submonolayer coverage) lying at the interface between palladium and thiolate moieties. ${ }^{5-7}$ Density functional theory (DFT) calculations show that the sulfide layer comes from the $\mathrm{S}-\mathrm{C}$ bond scission caused by a charge transfer from the palladium $\mathrm{d}$ band to antibonding thiolate orbitals. ${ }^{7}$ In addition, it was recently shown that the chemical composition of the surface of alkanethiolate-protected palladium nanoparticles is very similar to that of alkanethiolatemodified bulk palladium: $\operatorname{Pd}(0)$ cores are surrounded by a submonolayer of sulfide species, which are protected by alkanethiolates. $^{8-10}$ Despite the fact that these particles have very interesting properties that can be exploited in catalysis ${ }^{11}$ and sensing, ${ }^{12}$ their detailed structure has been barely studied. Indeed, even in recent studies, the already known ${ }^{5,8}$ chemical nature of the Pd-thiolate interface is ignored. ${ }^{11-14}$

It is well-known that thiolate species produce a reconstruction of the gold surface both in planar substrates ${ }^{15}$ and in nanoparticles. ${ }^{16}$ Results from molecular simulations suggest that the influence of the capping molecule can also reach deeper atomic layers in gold nanoparticles smaller than 2 $\mathrm{nm} .^{17,18}$ In the case of planar palladium, DFT calculations show that when only thiolates are adsorbed on the surface, there is no reordering of the metal atoms in the first layers; ${ }^{7,13}$ however, when sulfide is incorporated as an adsorbate, a considerable surface reconstruction takes place. This behavior is attributed to

Received: August 18, 2014

Revised: September 25, 2014

Published: September 29, 2014 
the strong bonding of sulfide species to palladium (binding energy $-5.0 /-5.6 \mathrm{eV}$ ) compared to thiolate moieties (binding energy $-2.7 /-3.2 \mathrm{eV})^{7}$

Regarding the structure of passivated palladium nanoparticles, there are differing reports where the metal atoms are found to have either a crystalline or an amorphous structure. In one of the first reported studies on thiolateprotected palladium nanoparticles with an amorphous structure, Hou et al. ${ }^{19,20}$ synthesized poly(vinylpyrrolidone) (PVP)-protected palladium nanoparticles using either a strong (sodium borohydride) or a weak (ethanol) reducing agent, which were subsequently modified with dodecanethiol. The authors found that the nanoparticles synthesized with the strong reducing agent had an amorphous structure, while the nanoparticles prepared with the weak reducing agent had a crystalline structure. They explained this difference by considering the reduction rate of the precursors to generate metallic palladium: with sodium borohydride, the reduction takes place very fast and the nanoparticles are produced without time to relax the structure, while, with ethanol, the reduction proceeds slowly and palladium atoms have enough time to diffuse and form ordered structures.

The crystallinity modulation of palladium nanoparticles by changing the protecting molecule was studied by highresolution transmission electron microscopy (HRTEM) and molecular dynamics (MD) simulations by Liu et al. ${ }^{21}$ It was found that while palladium atoms in nanoparticles prepared with oleylamine formed an ordered structure, the substitution of oleylamine by trioctylphosphine reduced the order considerably; besides, the particles recovered their crystalline structure when the surfactant was switched back. The difference is presumably due to the strength of the ligand-metal interactions, since palladium interacts with oleylamine less strongly than with trioctylphosphine. However, it has to be considered that in this study the MD simulations were carried out without an atomistic description of the ligands. In a more recent paper, the authors described their study of the sulfidization process of $6 \mathrm{~nm}$ oleylamine-protected palladium nanoparticles at high temperature. ${ }^{22}$ They found that by adding dodecanethiol the nanoparticles gradually transformed into $\mathrm{PdS}_{x}$ at temperatures above $363 \mathrm{~K}$. Below that temperature, the sulfidization was restricted to the surface and the nanoparticles showed a crystalline structure when observed by TEM.

In another work recently published, the synthesis of palladium nanoparticles protected with dodecyl sulfide (DDS), to be used as a catalyst for the synthesis of hydrogen peroxide, was reported. ${ }^{23}$ The particles had a crystalline structure clearly observed by HRTEM imaging. However, when DDS was exchanged with a thiol (mercaptoacetic acid), the metal atoms in the particles became disordered and the well-defined structure was lost. When the ligand is DDS, since the bond between sulfur and palladium is produced by the lone pair in sulfur, the energy of this interaction is weak. On the other hand, with the thiol, a strong covalent bond is produced with the metal; moreover, the formation of palladium sulfide is also possible, and in that case, the interaction is even stronger.

Finally, in a study of 11-mercaptoundecanoic acid-protected palladium nanoparticles by HRTEM, Cargnello et al. ${ }^{24}$ found that the crystallinity of the nanoparticles strongly depends on the temperature during the synthesis. It was observed that the lower the temperature used, the greater the crystallinity of the nanoparticles. They explained this behavior with the same arguments as Hou et al.; ${ }^{19,20}$ i.e., low temperatures decrease the reaction rates, which leads to an ordered packing of palladium atoms. However, it is also possible that the chemical nature of the ligand-metal bond changes at different temperatures.

In summary, the differences in the crystalline structure of palladium nanoparticles have been explained in different ways. The first explanation is based on the difference in the reduction rates of $\mathrm{Pd}$ (II) species to form the nanoparticles: fast reduction produces disordered structures, and slow reduction produces ordered metallic structures. The second explanation relates the difference in the ordering of the metal atoms with the strength of the ligand-metal interaction: the use of ligands which interact strongly with palladium produces amorphous structures, while the use of ligands which interact weakly produces ordered metallic structures. A third interpretation includes the diffusion of sulfide into the nanoparticle core.

In this paper we present results regarding the ordering of metal atoms both in thiolate-protected palladium nanoparticles (Pd@S-SR NPs) and in alkylamine-protected palladium nanoparticles ( $\left.\mathrm{Pd} @ \mathrm{NH}_{2} \mathrm{R} \mathrm{NPs}\right)$. The structure of the nanoparticles was investigated by aberration-corrected high-angle annular dark field scanning transmission electron microscopy (HAADF-STEM), and the interpretation of the images was done by contrasting the experimental results with predictions obtained by means of Langevin dynamics simulations, where both the metal and the ligands were modeled with atomistic detail.

\section{EXPERIMENTAL AND THEORETICAL METHODS}

Nanoparticle Synthesis. Pd@S-SR NPs were prepared by the two-phase Brust-Schiffrin method, ${ }^{25,26}$ and $\mathrm{Pd@NH} \mathrm{N}_{2} \mathrm{R}$ NPs were prepared by a modification of that method where amines are used in place of thiols. ${ }^{27}$ The average diameters $\langle D\rangle$ of the particles obtained were $3.3 \pm 0.9$ and $3.2 \pm 0.7 \mathrm{~nm}$, respectively. Also, thiolate-protected nanoparticles were prepared by ligand place exchange of $\mathrm{Pd} @ \mathrm{NH}_{2} \mathrm{R}$ NPs with thiol. Briefly, Pd@ $\mathrm{NH}_{2} \mathrm{R}$ NPs were placed in a solution of thiol (RSH:Pd $=1: 1$ ) and stirred for $24 \mathrm{~h}$ at room temperature to replace amine by thiolate on the nanoparticle surface. Although this procedure leads to partial ligand exchange, sulfide and thiolate were found as adsorbates, besides nitrogen species. Thus, we refer to them as Pd@ $\mathrm{NH}_{2} \mathrm{R}-\mathrm{S}$-SR NPs. The thiol used in this work was dodecanethiol and the amine dodecylamine. Details on the synthesis of the nanoparticles used and an extensive characterization of them can be found in a previous paper. $^{8}$

Aberration-Corrected Scanning Transmission Electron Microscopy. STEM was carried out with a JEOL JEMARM200F aberration-corrected microscope at the University of Texas at San Antonio, operating at $200 \mathrm{kV}$, equipped with a Schottky field emission gun (FEG), a hexapole spherical aberration (Cs) probe corrector (CEOS $\mathrm{GmbH}$ ), and an HAADF detector. The probe size used for acquiring the HAADF images was $0.095 \mathrm{~nm}$. The nanoparticle suspension was drop-cast on an amorphous-carbon-covered copper grid.

Langevin Dynamics Simulations. Langevin dynamics simulations were performed to study the adsorption of butylamine, sulfide, and butanethiolate onto palladium clusters. Metallic interactions were represented by means of the second moment approximation of the tight-binding (TB-SMA) potential: ${ }^{28,29}$

$$
U_{\mathrm{TB}-\mathrm{SMA}}=\sum_{i}\left(U_{\mathrm{R}}^{i}+U_{\mathrm{B}}^{i}\right)
$$


In this equation, $U_{\mathrm{B}}$ is the band energy, which takes into account the many-body character of metallic bonds

$$
U_{\mathrm{B}}^{i}=-\left\{\sum_{j} \xi_{\alpha \beta}{ }^{2} \exp \left[-2 q_{\alpha \beta}\left(r_{i j} / r_{0}^{\alpha \beta}-1\right)\right]\right\}^{-1 / 2}
$$

and $U_{R}$ is a pair term representing the repulsion between nuclei

$$
U_{\mathrm{R}}^{i}=\sum_{j} A_{\alpha \beta} \exp \left[-p_{\alpha \beta}\left(r_{i j} / r_{0}^{\alpha \beta}-1\right)\right]
$$

Parameters for the simulation of palladium were taken from ref 30.

Sulfide-metal interaction was represented with a Morse potential, parametrized for reproducing the adsorption of sulfide onto perfect planar palladium surfaces. Optimization of the site and energy of adsorption was accomplished by performing DFT calculations using the CASTEP code. ${ }^{31}$ The parameters obtained are $D_{\mathrm{e}}=1.2746 \mathrm{eV}, r_{\mathrm{e}}=2.2898 \AA$, and $\alpha=$ $1.47 \AA^{-1}$.

To represent the interaction between head groups of butanethiolate (thiolate groups) and palladium, the new semiempirical potential previously developed by the authors ${ }^{32}$ was parametrized by means of DFT calculations. The main characteristic of this potential is that bond orders (BOs) of the interacting species are considered to distinguish different geometries of adsorption using distinct parameters as a function of the coordination. The parameters obtained and bond orders are shown in Table 1.

Table 1. Parameters Obtained in the Fit of the Potential for the Palladium Thiolate Group

\begin{tabular}{lcccc} 
adsorption site & $D_{\mathrm{e}} / \mathrm{eV}$ & $r_{\mathrm{e}} / \AA$ & $\alpha / \AA^{-1}$ & $\mathrm{~S} \mathrm{BO}$ \\
on top & 1.006932 & 2.2515 & 1.47 & 1.000000 \\
bridge & 0.667994 & 2.4557 & 1.47 & 2.013076 \\
hollow fcc & 0.729014 & 2.3198 & 1.47 & 2.999774 \\
\hline
\end{tabular}

The representation of the alkyl chain was accomplished following the concepts of the universal force field (UFF) ${ }^{33}$ and within the united atom (UA) approximation. The parameters can be found in ref 32 .

Simulation of HAADF-STEM Images. The final configurations of the Langevin dynamics simulations were used as input coordinates for the simulation of HAADF-STEM. For these simulations we used the multislice method as implemented in the XHREM package by Ishizuka that uses an algorithm based on fast Fourier transforms (FFTs). In simulated HAADF-STEM, the particle acting as a sample was dissected into 20 slices (in the direction of the electron beam), each slice treated independently through an averaged projected potential. In accordance with the experimental STEM images used in this study, the simulated microscope was set at an electron beam voltage of $200 \mathrm{kV}$, with a resolution close to 0.1 $\mathrm{nm}$.

\section{RESULTS AND DISCUSSION}

A direct comparison between the aberration-corrected HAADF-STEM images of the first two kinds of particles shows a clear difference in crystallinity (Figure 1). While amineprotected palladium nanoparticles have a crystalline structure (Figure 1a), it was not possible to distinguish lattice fringes in any of the several images of thiolate-protected palladium nanoparticles (Figure 1c). This is clear evidence that the crystalline structure of the particles is largely affected by the capping agent: a crystalline structure in amine-protected particles and a disordered structure in thiolate-protected particles. More images are included in the Supporting Information (Figures S1 and S2). The FFT of the highresolution images of Figure la,c show even more clearly the differences in crystallinity. The FFT shown in Figure $1 \mathrm{~b}$ is typical of a particle with a 5-fold symmetry, whereas in the FFT in Figure 1d it is not possible to locate spots that correspond to a particular set of atomic planes. From the images it is not possible to determine with total certainty whether the whole particle has an amorphous structure or only the surface is distorted while the core of the particle retains the crystalline structure of metallic palladium. We will discuss this point later in relation to the Langevin dynamics simulations and the simulation of the HAADF-STEM images.

To determine if the growing conditions of the nanoparticles, i.e., the reduction rate, the presence of thiolate in the reaction media, etc., were responsible for the amorphous structure, thiolate-protected palladium nanoparticles prepared by ligand place exchange were also studied. We have reported that the alkylamines were partially exchanged by dodecanethiol molecules, resulting in particles with a mixed capping agent. X-ray photoelectron spectroscopy (XPS) data showed that the relative surface amount of sulfide and thiolate species on the $\mathrm{Pd} @ \mathrm{NH}_{2} \mathrm{R}-\mathrm{S}-\mathrm{SR}$ NPs was comparable to that on the Pd@S-SR NPs (thiolate:sulfide $\approx 1: 1$ ), although the total sulfide + thiolate quantity was lower ( (sulfide + thiolate $): P d \approx 0.3: 1)$. As can be observed in Figure 1e,f these nanoparticles show an amorphous structure, similar to that of the thiolate-protected nanoparticles prepared by the Brust-Schiffrin method (Figure $1 c, d)$. Notably, approximately $40-50 \%$ of the nanoparticles showed a crystalline structure, although the surface was always distorted (see Figure S3, Supporting Information). Probably this was caused by the incomplete ligand exchange. All in all, it is remarkable that the Pd@NH $\mathrm{N}_{2} \mathrm{R}-\mathrm{S}-\mathrm{SR}$ NPs showed a distorted or completely amorphous structure even when the total amount of thiolate + sulfide measured was lower than in the case of Pd@S-SR NPs ((sulfide + thiolate $): P d \approx 0.7: 1){ }^{8}$ This result rules out the possibility that the reaction rate can be responsible for the differences in the crystallinity of the nanoparticles. Also, it strongly supports the idea that the surface chemistry of the nanoparticle affects the arrangement of the atoms in the whole particle.

To investigate how the amount of a particular ligand affects the structural properties of a nanoparticle, we performed Langevin dynamics simulations on a set of palladium nanoparticles with different shapes, sizes, and compositions of the capping layer. Details on the method used can be found in the Supporting Information. Three different particle morphologies were considered to study the difference between monocrystalline and twinned nanoparticles: octahedra (Oh; face-centered cubic (fcc) structure), icosahedra (Ih; noncompact structure), and Marks decahedra (Dh; noncompact structure). The number of metal atoms on each particle is directly related to the geometry. The complete set of particles considered in the study was $\mathrm{Ih}_{561}, \mathrm{Ih}_{923}, \mathrm{Oh}_{489}, \mathrm{Oh}_{891}$, and $\mathrm{Dh}_{584}$. The model particles were protected with a capping layer made of a mixture of sulfide (S) and alkanethiolate (SR). Butanethiolate was chosen to consider the interaction between hydrocarbon chains, which are negligible in alkanethiols with a lower number of carbon atoms. For each particle, the total number of ligands (sulfide plus butanethiolate) was fixed, but 

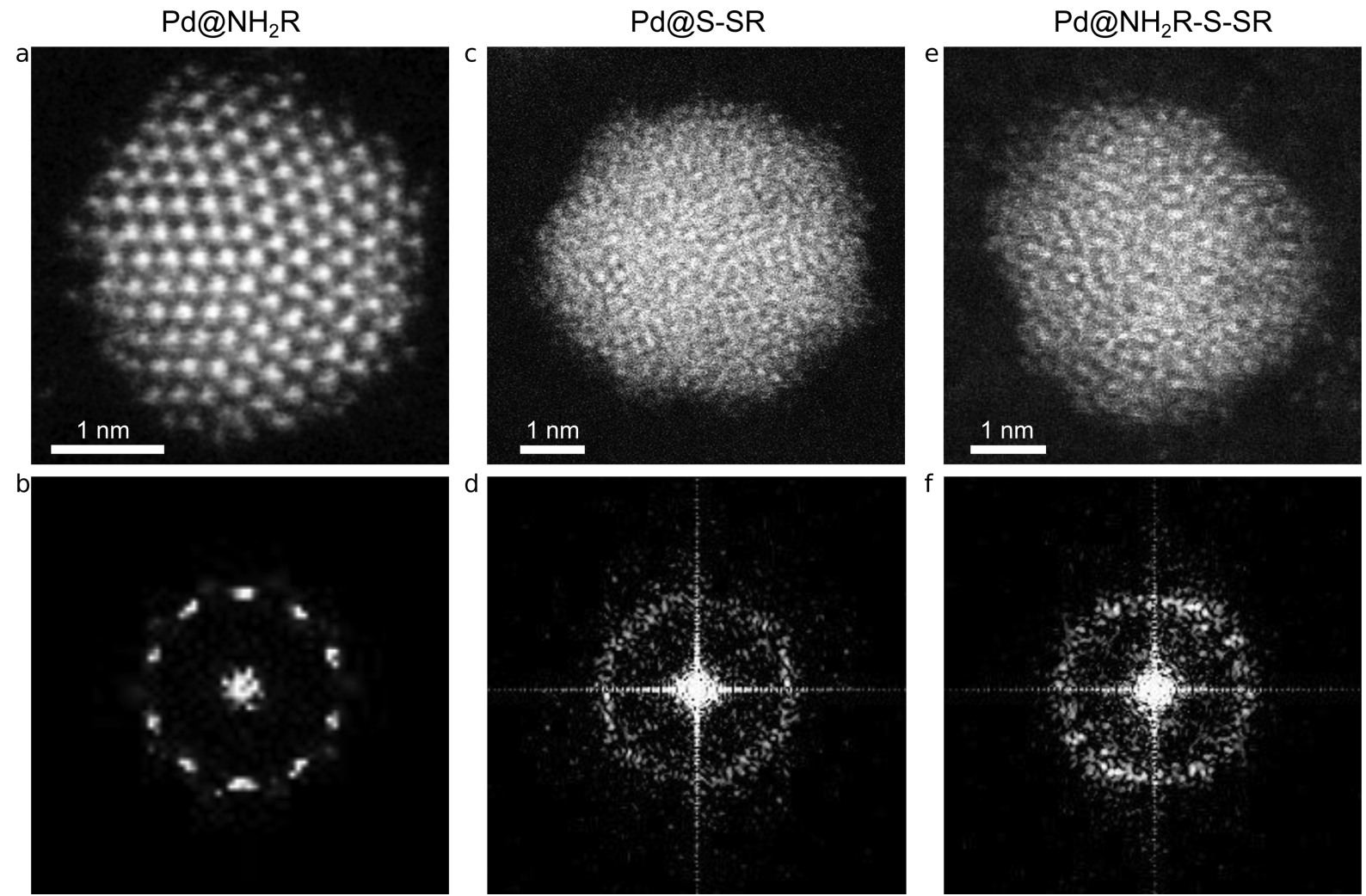

Figure 1. Representative aberration-corrected HAADF-STEM images of the three kinds of Pd nanoparticles studied: (a) dodecylamine-protected palladium nanoparticles, (b) FFT of (a), (c) dodecanethiolate-protected palladium nanoparticles, (d) FFT of (c), (e) dodecanethiolate-protected palladium nanoparticles prepared by ligand place exchange of dodecylamine-protected palladium nanoparticles with dodecanethiol, (f) FFT of (e).
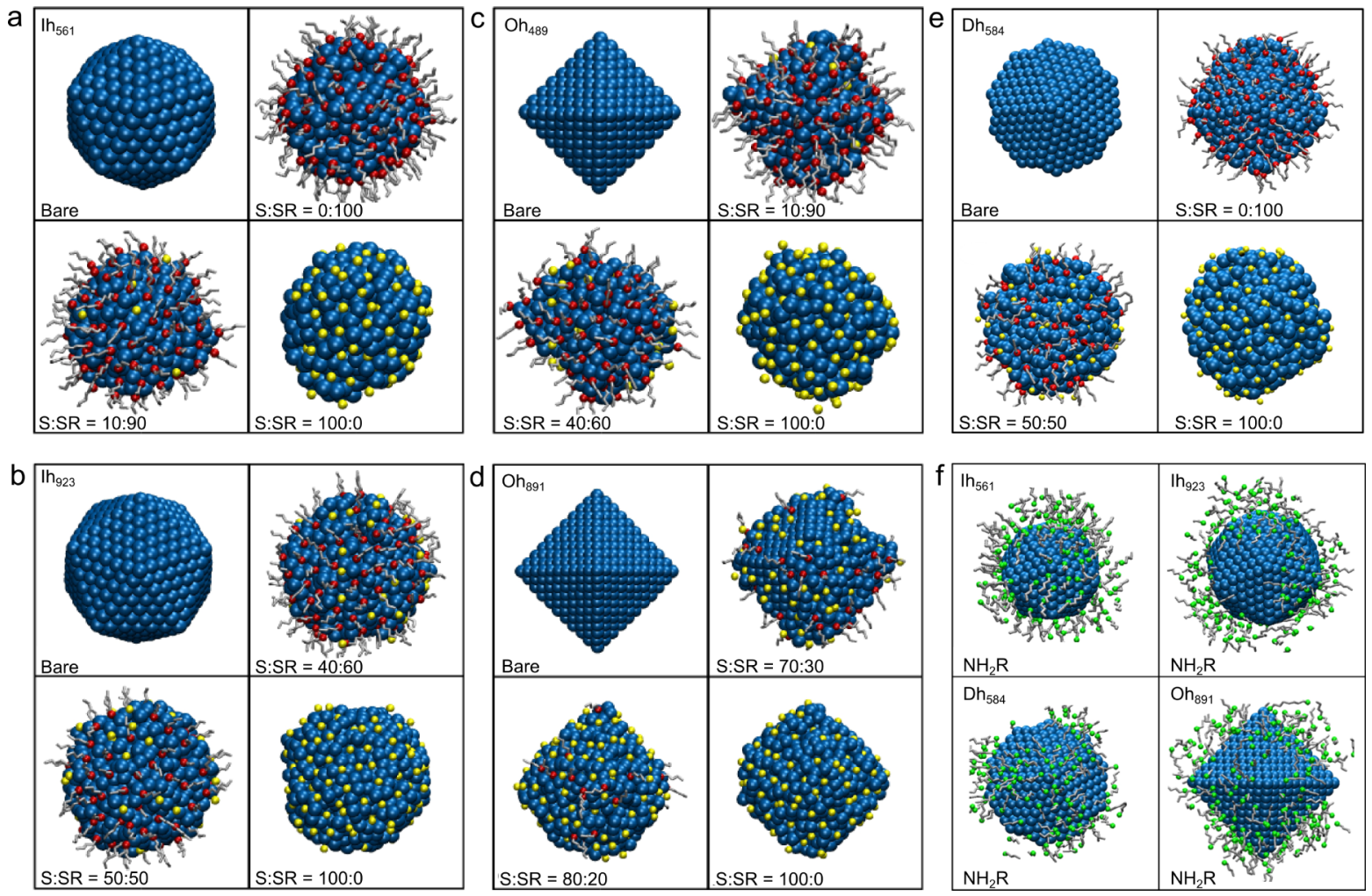

Figure 2. Configuration of the palladium nanoparticles studied (blue, palladium atoms; yellow, sulfide; red, thiolate group; gray, alkyl chains; green, amine group): (a-e) Pd nanoparticles passivated with sulfide:thiolate ( $\mathrm{S}: \mathrm{SR}$ ) in different percentages, (f) Pd nanoparticles passivated with alkylamines. The images are not on the same scale. 


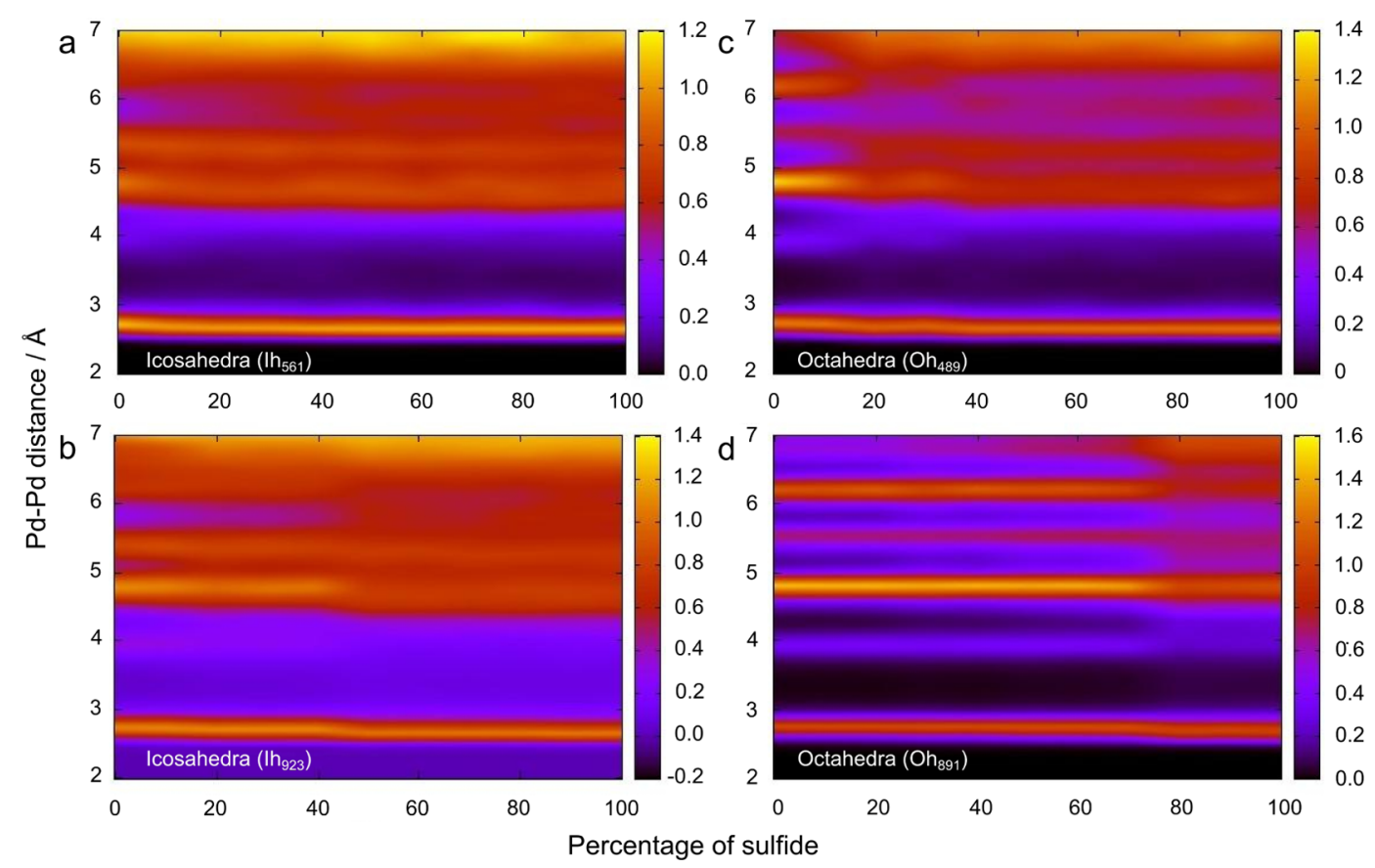

Figure 3. Color maps representing the pair distribution functions of palladium atoms in nanoparticles passivated with different percentages of sulfide (the sum of sulfide and thiolate equals $100 \%$ ). Icosahedra: (a) $\mathrm{Ih}_{561}$, (b) $\mathrm{Ih}_{923}$. Octahedra: (c) $\mathrm{Oh}_{489}$, (d) $\mathrm{Oh}_{891}$. The color scale on the right represents the value of $g_{r}$.

several proportions of the capping species were considered in the simulations. Also, palladium nanoparticles protected with amines $\left(\mathrm{NH}_{2} \mathrm{R}\right)$ were studied. In that case, butylamine was the chosen compound.

Each metal nanoparticle was thermalized and then embedded in amines or in a mixture of sulfide and thiols in a particular proportion. Two nanoseconds of Langevin dynamics was performed, allowing the ligands to passivate the particle. Several final configurations of the runs are shown in Figure 2. As can be immediately noted, after passivation, the structure is lost to some degree in all cases for sulfide- and thiolate-protected particles; the smaller the particle, the stronger the effect. If adsorbates are only thiolates, the loss of order is smaller than when there is some amount of sulfide in the system, and the greater the proportion of sulfide, the stronger the disorder in the nanoparticles. The effect is also larger in particles that lack a compact structure. For the largest particles with an fcc structure (Oh), only high concentrations of sulfide produce an appreciable structural deformation. In the case of amineprotected nanoparticles, the structures do not show appreciable changes (Figure 2f).

To measure how the percentage of sulfide affects the order in the metallic cores, the pair distribution function $\left(g_{\mathrm{r}}\right)$ of palladium atoms was calculated. Several calculated $g_{\mathrm{r}}$ functions, represented as color maps, are shown in Figure 3. For compact fcc structures, as Oh, a higher proportion of sulfide is necessary to observe the loss of order, in comparison with Ih nanoparticles of similar size. As can be observed in Figure 3d, $\mathrm{Oh}_{891}$ is a system that conserves its structure even with a high percentage of sulfide; this can be deduced by the position of the peaks corresponding to the first, second, and subsequent neighbors: the peaks remain stable up to a sulfide amount of $70-75 \%$. In the case of noncompact particles (Ih) or the smaller Oh, the loss of structure can be observed with smaller quantities of sulfide in the medium. The analysis of $g_{r}$ at different distances from the center of mass of the nanoparticle helps to analyze the radial location of the disorder, i.e., if the whole particle is affected by the ligand or only the outer layers are influenced. $g_{\mathrm{r}}$ revealed different results for different particle sizes, but in general, it can be observed that, for sulfide-thiolate capping, almost all the metallic particle is changed by the presence of the protecting layer (see Figures S5-S8, Supporting Information). It can be concluded that, in addition to the role of size (smaller systems have larger surface/volume ratios and, thus, are exposed to smaller cohesive forces), the crystal structure of the particle before passivation has an influence on the conservation of order after the addition of the ligands.

It is also important to establish if sulfur diffuses or preexists in the interior of the nanoparticle in thiolated nanoparticles. It can be argued that, during nanoparticle growth in the presence of thiol molecules, the growing $\operatorname{Pd}(0)$ cores can produce sulfide that can be trapped inside the nanoparticles and, hence, produce amorphous $\mathrm{PdS}_{x}$ nanoparticles. However, there are several pieces of evidence in favor of the idea that sulfide is superficial and the strong $\mathrm{Pd}-\mathrm{S}$ bond also has an effect in the nanoparticle core. First, we have shown that palladium nanoparticles prepared by ligand place exchange of previously prepared amine-protected particles also showed an amorphous structure (Figure 1e). Second, although it can be observed in the slice of the nanoparticles (Figure S4, Supporting Information) that there is some diffusion of sulfide into the interior of the nanoparticles, the sulfur is preferentially located on the surface of the nanoparticles with significant abundance between the two first atomic layers. Third, all this is in accordance with the results from XPS experiments published in a previous paper. ${ }^{8}$ There, it was shown that the total S:Pd atomic ratios in thiolate-protected nanoparticles measured by XPS were similar when the X-ray source energy was set to 250 $\mathrm{eV}$ (analyzed depth $\sim 0.5 \mathrm{~nm}$ ) or $1253.6 \mathrm{eV}$ (analyzed depth $\sim 2.5 \mathrm{~nm}$ ). These results prove that diffusion of sulfide into the nanoparticle core, producing a $\mathrm{PdS}_{x}$ nanoparticle in the whole 


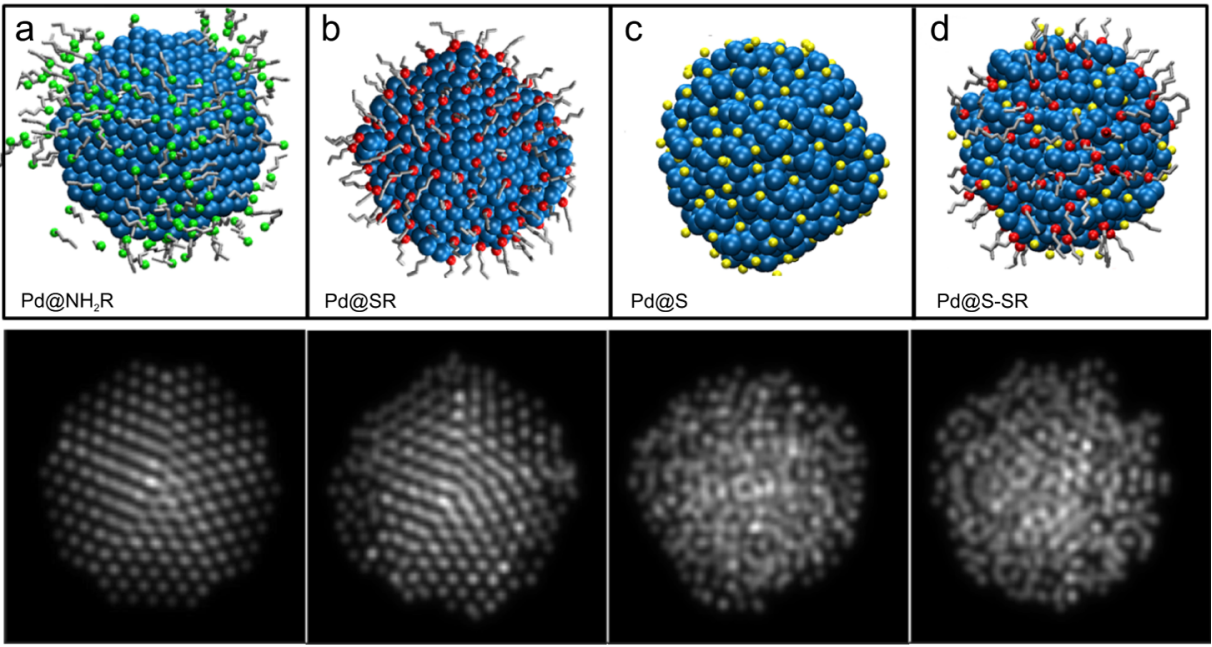

Figure 4. Comparison of the HAADF-STEM image simulation of the Marks decahedral palladium nanoparticles protected with different ligands: (a) butylamine $\left(\mathrm{NH}_{2} \mathrm{R}\right)$, (b) butanethiolate (SR), (c) sulfide (S), (d) sulfide + butanethiolate (S-SR).

particle, is not necessary to produce amorphous palladium nanoparticles with a size of $\sim 3 \mathrm{~nm}$.

The final configurations of the Langevin dynamics simulations were used to generate simulated HAADF-STEM images to compare theoretical and experimental results. The simulated images for decahedral palladium particles protected with different ligands are shown in Figure 4. For amineprotected particles, the atomic columns are clearly observed with the particle oriented on the 5-fold rotation axis (Figure $4 a)$. When thiolate alone is used as a ligand, only a disorder in the layers near the surface is observed in the images (Figure $4 b)$. The order in the structure is lost when sulfide is the capping agent, as expected from the previously stated results. The whole particle shows a disordered structure (Figure 4c). The same is obtained when a mixture of sulfide and thiolate is incorporated as a protecting layer (Figure $4 \mathrm{~d}$ ). These results support the interpretation of the experimental HAADF-STEM images in terms of the models proposed by the simulations and strengthen the notion that mainly sulfide is responsible for the induced disorder in the particles.

\section{CONCLUSIONS}

Considering all the discussed results, it can be concluded that thiolate-protected palladium nanoparticles $\sim 3 \mathrm{~nm}$ in size prepared at room temperature show an amorphous structure. Contrary to $\sim 6 \mathrm{~nm}$ nanoparticles where high-temperatureassisted diffusion of sulfide into the nanoparticles is needed to disorder the palladium atoms in the whole nanoparticle, ${ }^{22}$ in the case of $3 \mathrm{~nm}$ thiolate-protected palladium nanoparticles, amorphous structures are observed at room temperature, produced by the strong $\mathrm{Pd}-\mathrm{S}$ interaction on the nanoparticle surface. From the Langevin dynamics simulations it was observed that the loss of an ordered structure after passivation of palladium nanoparticles depends on the quantity of sulfide on the particle surface. The sulfide comes from the breaking of the $\mathrm{S}-\mathrm{C}$ bond of alkanethiols upon their adsorption on palladium clusters and is placed between the first palladium layers to form a passivated interface. In the case of noncompact structures, such as icosahedra and decahedra, the effect is stronger than in compact fcc structures due to the lower cohesive energy of the metal system. A larger amount of sulfide is necessary in the latter case to observe a comparable loss of order. These results show that the crystallinity of $\sim 3 \mathrm{~nm}$ thiolate-protected palladium nanoparticles is remarkably different from that of gold nanoparticles of similar size and a capping agent, a behavior explained in terms of the difference in the surface chemistry of both systems.

\section{ASSOCIATED CONTENT}

\section{S Supporting Information}

Plots of the radial distribution function, HAADF-STEM images, and diagram of a slice of the nanoparticle showing the diffusion of sulfur. This material is available free of charge via the Internet at http://pubs.acs.org.

\section{AUTHOR INFORMATION}

\section{Corresponding Authors}

*E-mail: gcorthey@inifta.unlp.edu.ar.

*E-mail: marmariscal@fcq.unc.edu.ar.

*E-mail: mfonti@inifta.unlp.edu.ar.

\section{Present Addresses}

${ }^{\nabla}$ Max Planck Institute for the Structure and Dynamics of Matter, Luruper Chaussee 149, 22761 Hamburg, Germany.

Ophysics Department, University of Trieste, Strada Costiera 11, I-34151 Trieste, Italy.

UOW Electron Microscopy Centre, University of Wollongong, Wollongong, New South Wales 2500, Australia.

\section{Author Contributions}

\#G.C. and J.A.O.-A. contributed equally to this work.

\section{Notes}

The authors declare no competing financial interest.

\section{ACKNOWLEDGMENTS}

We acknowledge financial support from the Agencia Nacional de Promoción Científica y Tecnológica (Grants PICT 20101233, PICT 2010-0423, PICT 2006-621, and PICT 2010-2554 and Nanotechnology Network PAE22711), CONICET (PIP Grants 11220090100139 and 112200801000983), the Universidad Nacional de La Plata (UNLP), the Universidad Nacional de Cordoba, Argentina, The Welch Foundation Agency (Project AX-1615: Controlling the Shape and Particles Using Wet Chemistry Methods and Its Application to Synthesis of Hollow Bimetallic Nanostructures), the National Science 
Foundation (NSF) (PREM Grant DMR-0934218, NSF Grant 1103730: Alloys at the Nanoscale; The Case of Nanoparticles Second Phase), and CONACYT, México (Grant CIAM 148967). We acknowledge the Texas Advanced Computing Center (TACC) and the Sistema Nacional de Computacion de Alto Desempeño (SNCAD). G.C. thanks the Swiss National Science Foundation and UNLP for financial support. We thank Eugenia Zelaya (Centro Atómico Bariloche, Argentina) for several important discussions and preliminary TEM experiments.

\section{REFERENCES}

(1) Love, J.; Estroff, L.; Kriebel, J.; Nuzzo, R.; Whitesides, G. SelfAssembled Monolayers of Thiolates on Metals as a Form of Nanotechnology. Chem. Rev. 2005, 105, 1103-1170.

(2) Pensa, E.; Cortés, E.; Corthey, G.; Carro, P.; Vericat, C.; Fonticelli, M. H.; Benitez, G.; Rubert, A. A.; Salvarezza, R. C. The Chemistry of the Sulfur-Gold Interface: In Search of a Unified Model. Acc. Chem. Res. 2012, 45, 1183-1192.

(3) Häkkinen, H. The Gold-Sulfur Interface at the Nanoscale. Nat. Chem. 2012, 4, 443-455.

(4) Azcárate, J. C.; Corthey, G.; Pensa, E.; Vericat, C.; Fonticelli, M. H.; Salvarezza, R. C.; Carro, P. Understanding the Surface Chemistry of Thiolate-Protected Metallic Nanoparticles. J. Phys. Chem. Lett. 2013, 4, 3127-3138.

(5) Love, J.; Wolfe, D.; Haasch, R.; Chabinyc, M.; Paul, K.; Whitesides, G.; Nuzzo, R. Formation and Structure of Self-Assembled Monolayers of Alkanethiolates on Palladium. J. Am. Chem. Soc. 2003, 125, 2597-2609.

(6) Corthey, G.; Rubert, A. A.; Benitez, G. A.; Fonticelli, M. H.; Salvarezza, R. C. Electrochemical and X-ray Photoelectron Spectroscopy Characterization of Alkanethiols Adsorbed on Palladium Surfaces. J. Phys. Chem. C 2009, 113, 6735-6742.

(7) Carro, P.; Corthey, G.; Rubert, A. A.; Benitez, G. A.; Fonticelli, M. H.; Salvarezza, R. C. The Complex Thiol-Palladium Interface: A Theoretical and Experimental Study. Langmuir 2010, 26, 1465514662.

(8) Corthey, G.; Rubert, A. A.; Picone, A. L.; Casillas, G.; Giovanetti, L. J.; Ramallo-López, J. M.; Zelaya, E.; Benitez, G. A.; Requejo, F. G.; José-Yacamán, M.; et al. New Insights into the Chemistry of ThiolateProtected Palladium Nanoparticles. J. Phys. Chem. C 2012, 116, 98309837.

(9) Murayama, H.; Ichikuni, N.; Negishi, Y.; Nagata, T.; Tsukuda, T. EXAFS Study on Interfacial Structure between Pd Cluster and nOctadecanethiolate Monolayer: Formation of Mixed Pd-S Interlayer. Chem. Phys. Lett. 2003, 376, 26-32.

(10) Litrán, R.; Sampedro, B.; Rojas, T. C.; Multigner, M.; SánchezLópez, J. C.; Crespo, P.; López-Cartes, C.; García, M. A.; Hernando, A.; Fernández, A. Magnetic and Microstructural Analysis of Palladium Nanoparticles with Different Capping Systems. Phys. Rev. B 2006, 73, 054404.

(11) Marshall, S. T.; O’Brien, M.; Oetter, B.; Corpuz, A.; Richards, R. M.; Schwartz, D. K.; Medlin, J. W. Controlled Selectivity for Palladium Catalysts Using Self-Assembled Monolayers. Nat. Mater. 2010, 9, 853-858.

(12) Moreno, M.; Ibañez, F. J.; Jasinski, J. B.; Zamborini, F. P. Hydrogen Reactivity of Palladium Nanoparticles Coated with Mixed Monolayers of Alkyl Thiols and Alkyl Amines for Sensing and Catalysis Applications. J. Am. Chem. Soc. 2011, 133, 4389-4397.

(13) Majumder, C. Adsorption of Thiols on the Pd(111) Surface: A

First Principles Study. Langmuir 2008, 24, 10838-10842.

(14) Fresch, B.; Remacle, F. Tuning the Properties of Pd Nanoclusters by Ligand Coatings: Electronic Structure Computations on Phosphine, Thiol, and Mixed Phosphine-Thiol Ligand Shells. J. Phys. Chem. C 2014, 118, 9790-9800.

(15) Cossaro, A.; Mazzarello, R.; Rousseau, R.; Casalis, L.; Verdini, A.; Kohlmeyer, A.; Floreano, L.; Scandolo, S.; Morgante, A.; Klein, M.
L.; et al. X-ray Diffraction and Computation Yield the Structure of Alkanethiols on Gold(111). Science 2008, 321, 943-946.

(16) Jadzinsky, P. D.; Calero, G.; Ackerson, C. J.; Bushnell, D. A.; Kornberg, R. D. Structure of a Thiol Monolayer-Protected Gold Nanoparticle at $1.1 \AA$ Resolution. Science 2007, 318, 430-433.

(17) Mariscal, M. M.; Olmos-Asar, J. A.; Gutierrez-Wing, C.; Mayoral, A.; Yacaman, M. J. On the Atomic Structure of ThiolProtected Gold Nanoparticles: A Combined Experimental and Theoretical Study. Phys. Chem. Chem. Phys. 2010, 12, 11785-11790.

(18) Olmos-Asar, J. A.; Ludueña, M.; Mariscal, M. M. Monolayer Protected Gold Nanoparticles. Effect of the Headgroup-Au Interaction. Phys. Chem. Chem. Phys. 2014, 16, 15979-15987.

(19) Lu, W.; Wang, B.; Wang, K.; Wang, X.; Hou, J. G. Synthesis and Characterization of Crystalline and Amorphous Palladium Nanoparticles. Langmuir 2003, 19, 5887-5891.

(20) Hou, J. G.; Wang, B.; Yang, J.; Wang, K.; Lu, W.; Li, Z.; Wang, H.; Chen, D. M.; Zhu, Q. Disorder and Suppression of Quantum Confinement Effects in Pd Nanoparticles. Phys. Rev. Lett. 2003, 90, 246803.

(21) Liu, Y.; Wang, C.; Wei, Y.; Zhu, L.; Li, D.; Jiang, J. S.; Markovic, N. M.; Stamenkovic, V. R.; Sun, S. Surfactant-Induced Postsynthetic Modulation of Pd Nanoparticle Crystallinity. Nano Lett. 2011, 11, 1614-1617.

(22) Liu, Y.; Sun, C.; Bolin, T.; Wu, T.; Liu, Y.; Sternberg, M.; Sun, S.; Lin, X.-M. Kinetic Pathway of Palladium Nanoparticle Sulfidation Process at High Temperatures. Nano Lett. 2013, 13, 4893-4901.

(23) Kim, J.-H.; Park, J.-S.; Chung, H.-W.; Boote, B. W.; Lee, T. R. Palladium Nanoshells Coated with Self-Assembled Monolayers and Their Catalytic Properties. RSC Adv. 2012, 2, 3968-3977.

(24) Cargnello, M.; Wieder, N. L.; Canton, P.; Montini, T.; Giambastiani, G.; Benedetti, A.; Gorte, R. J.; Fornasiero, P. A Versatile Approach to the Synthesis of Functionalized Thiol-Protected Palladium Nanoparticles. Chem. Mater. 2011, 23, 3961-3969.

(25) Brust, M.; Walker, M.; Bethell, D.; Schiffrin, D. J.; Whyman, R. Synthesis of Thiol-Derivatised Gold Nanoparticles in a Two-Phase Liquid-Liquid System. J. Chem. Soc., Chem. Commun. 1994, 801-802.

(26) Zamborini, F.; Gross, S.; Murray, R. Synthesis, Characterization, Reactivity, and Electrochemistry of Palladium Monolayer Protected Clusters. Langmuir 2001, 17, 481-488.

(27) Leff, D. V.; Brandt, L.; Heath, J. R. Synthesis and Characterization of Hydrophobic, Organically-Soluble Gold Nanocrystals Functionalized with Primary Amines. Langmuir 1996, 12, 4723-4730.

(28) Gupta, R. P. Lattice Relaxation at a Metal Surface. Phys. Rev. B 1981, 23, 6265-6270.

(29) Cleri, F.; Rosato, V. Tight-Binding Potentials for Transition Metals and Alloys. Phys. Rev. B 1993, 48, 22-33.

(30) Pittaway, F.; Paz-Borbón, L. O.; Johnston, R. L.; Arslan, H.; Ferrando, R.; Mottet, C.; Barcaro, G.; Fortunelli, A. Theoretical Studies of Palladium-Gold Nanoclusters: Pd-Au Clusters with up to 50 Atoms. J. Phys. Chem. C 2009, 113, 9141-9152.

(31) Clark, S. J.; Segall, M. D.; Pickard, C. J.; Hasnip, P. J.; Probert, M. I. J.; Refson, K.; Payne, M. C. First Principles Methods Using CASTEP. Z. Kristallogr. 2005, 220, 567-570.

(32) Olmos-Asar, J. A.; Rapallo, A.; Mariscal, M. M. Development of a Semiempirical Potential for Simulations of Thiol-Gold Interfaces. Application to Thiol-Protected Gold Nanoparticles. Phys. Chem. Chem. Phys. 2011, 13, 6500-6506.

(33) Rappe, A. K.; Casewit, C. J.; Colwell, K. S.; Goddard, W. A.; Skiff, W. M. UFF, a Full Periodic Table Force Field for Molecular Mechanics and Molecular Dynamics Simulations. J. Am. Chem. Soc. 1992, 114, 10024-10035. 